\title{
Optimal Placement and Sizing of the Storage Supporting Transmission and Distribution Networks
}

\author{
Mahdi Motalleb, Ehsan Reihani, Reza Ghorbani ${ }^{1}$
}

\begin{abstract}
Developments of renewable energy resources imposes many uncertainties and variabilities in power grids. One of the best approaches to mitigate these stochastic disturbances is thought the use of Battery Energy Storage System (BESS). Besides application of the BESS such as decreasing the disturbances in distribution system, the grid frequency can be controlled in contingencies using the appropriate storage in transmission network to compensate the power shortage. Thus, the optimal siting and sizing of the BESS is important to have the minimum costs and losses. This paper describes a heuristic method to find the optimal location(s) and capacity of a multi-purpose BESS including transmission and distribution parts. In the transmission storage part, a sensitive analysis is performed using Complex-Valued Neural Networks (CVNN) and Time Domain Power Flow (TDPF) in order to detect the optimal BESS location(s). Additionally, running TDPF and Economic Dispatch (ED) leads to the optimal BESS size. In the distribution storage part, the optimal BESS size is calculated to perform distribution grid services such as peak load shaving and load curve smoothing. The proposed method has been applied to a real model (Maui island in Hawai'i -United States) to calculate the optimal results for both transmission and distribution sides.
\end{abstract} (ED)

Index Terms - Battery Energy Storage System (BESS), Optimal Location, Optimal Capacity, Economic Dispatch

NOMENCLATURE

TDPF Formulation:

$I_{B u s} \quad$ Injected current vector to the buses which is positive in generation buses (PV-buses and slack bus) and negative in loads (PQ-buses).

$Y_{\text {Bus }} \quad$ Admittance matrix of the grid

$23 V_{\text {Bus }} \quad$ Voltage vector of the buses

$24 f, g$ Algebraic and differential equations to solve time domain problem

$25 f_{n} \quad$ A function dependent on the method of the integration

$26 \quad A_{c}^{i} \quad$ A matrix dependent on the algebraic and state Jacobian matrices

$27 x^{i+1}, y^{i+1}$ Calculated values of $x$ and $y$ in time $t=i+1$

ED Formulation:

$32 C_{G E N, i}^{\text {local }}$ Generation cost of the $i^{\text {th }}$ local generator $(\$ / h r)$

$33 C_{G E N, j}^{d i s t}$ Generation cost of the $j^{\text {th }}$ distant generator $(\$ / h r)$

\footnotetext{
${ }^{1}$ Mahdi Motalleb, Ehsan Reihani, and Reza Ghorbani, are with Renewable Energy Design Laboratory (REDLab) at the Department of Mechanical Engineering, University of Hawai'i Manoa, Honolulu, Hawai'i, 96822. Phone: +1-808-956-2292, Fax: +1808-956-0767. E-mail: motalleb@hawaii.edu, ereihani@hawaii.edu, rezag@hawaii.edu.
} 
$34 C_{B E S S, k}^{\text {total }}$ Total generation cost of the $k^{\text {th }}$ BESS (located in the $k^{\text {th }}$ sensitive bus) $(\$ / h r)$

35 NGlocal Number of the local generators (located in the sensitive buses)

36 NGdist Number of the generators located far from than the sensitive buses

37 NBESS Number of the required BESS stations (is equal to the number of the sensitive buses)

$38 a_{\text {local }, i}$ The first coefficient of the quadratic cost function of the $i^{\text {th }}$ local generator

$39 b_{\text {local }, i}$ The second coefficient of the quadratic cost function of the $i^{\text {th }}$ local generator

$40 c_{\text {local }, i}$ The third coefficient of the quadratic cost function of the $i^{\text {th }}$ local generator

$41 a_{\text {dist }, j}$ The first coefficient of the quadratic cost function of the $j^{\text {th }}$ distant generator

$42 b_{\text {dist }, j}$ The second coefficient of the quadratic cost function of the $j^{\text {th }}$ distant generator

$43 c_{d i s t, j}$ The third coefficient of the quadratic cost function of the $j^{\text {th }}$ distant generator

$44 P_{G E N, i}^{\text {local }}$ Generated power of the $i^{\text {th }}$ local generator $(M W)$

$45 P_{G E N, j}^{d i s t}$ Generated power of the $j^{\text {th }}$ distant generator $(M W)$

$46 C_{j, l}^{\text {trans }} \quad$ Power transmission cost between the $j^{\text {th }}$ and $l^{\text {th }}$ bus

$47 C_{B E S S, k}^{s t}$ Cost of storage unit in the $k^{\text {th }} \operatorname{BESS}(\$ / h r)$

$48 C_{B E S S, k}^{p c s}$ Cost of power conversion in the $k^{\text {th }} \operatorname{BESS}(\$ / h r)$

$49 \quad R_{j, l} \quad$ Resistance between $j^{\text {th }}$ and $l^{\text {th }}$ bus $(\Omega)$

$50 \quad X_{j, l}$ Reactance between $j^{\text {th }}$ and $l^{\text {th }}$ bus $(\Omega)$

$51 I_{j, l} \quad$ Passing current from $j^{\text {th }}$ to $l^{\text {th }}$ bus $(k A)$

$52 P_{j, l}^{\text {flow }}$ Transmitted active power from $j^{\text {th }}$ to $l^{\text {th }}$ bus $(M W)$

$53 V_{j, l} \quad$ Voltage difference between $j^{\text {th }}$ and $l^{\text {th }}$ bus $(\mathrm{kV})$

$54 C_{B E S S, k}^{s t, u n i t}$ Unit cost of the storage in the $k^{\text {th }}$ BESS ( $\left.\$ / M W h r\right)$

$55 P_{B E S S, k}$ Generated power of the $k^{\text {th }}$ BESS (located in the $k^{\text {th }}$ sensitive bus) $(M W)$

$56 C_{B E S S, k}^{p c s, u n t} \quad$ Unit cost of the power conversion in the $k^{\text {th }} \operatorname{BESS}(\$ / M W h r)$

$57 P_{G E N \text { min, } i}^{\text {local }}$ The minimum allowed power generation of the $i^{\text {th }}$ local generator $(M W)$

$58 P_{G E N \text { max }, i}^{\text {local }}$ The maximum allowed power generation of the $i^{\text {th }}$ local generator $(M W)$

$59 P_{G E N \min , j}^{d i s t}$ The minimum allowed power generation of the $j^{\text {th }}$ distant generator $(M W)$

$60 P_{G E N \max , j}^{\text {dist }}$ The maximum allowed power generation of the $j^{\text {th }}$ distant generator $(M W)$

$61 P_{r e q, m} \quad$ The required power for the $m^{\text {th }}$ sensitive bus to cope with the contingency $(M W)$

62

63 Peak Shaving:

$64 P_{b a t}(t)$ Battery power flow at time $t(\mathrm{~kW})$

$65 P_{c i r}(t)$ Circuit power demand at time $t(k W)$

$66 S O C_{\min }$ The minimum allowed state of charge of BESS 
$S O C_{\max }$ The maximum allowed state of charge of BESS

$E_{\text {tot }} \quad$ Total capacity of distribution part of BESS $(k W h)$

$E(t)$ Energy level of distribution part of BESS at time $t(k W h)$

$\Delta t \quad$ Optimization time interval $(h r)$

$P_{r e f}(t)$ Reference power curve at time $t(k W)$

\section{Load Curve Smoothing:}

\section{$P_{L}(t)$ Smoothing power line $(k W)$}

$t_{k} \quad$ The $k^{\text {th }}$ time step

\section{INTRODUCTION}

High penetration of renewable energy generation poses some challenges in the operation of power systems. The main issue inherent with renewables is intermittency of power which will cause fluctuations in voltage and frequency of the power grid. BESS is one of the leading means to solve problems caused by these fluctuations. Applications of BESS have other benefits in the power grid such as frequency control, reserve providing, and flattening the load curve. The capacity and location of the BESS are important for costeffectiveness of storage applications. Allocation of BESS in the appropriate locations increase losses in the grid [1].

This paper proposes a framework to optimize the location(s) and size of the BESS for two purposes including: decreasing the fluctuations from the renewable energy sources in the distribution side, and controlling the frequency under contingencies in the transmission side. The transmission BESS compensate for the power shortage in the abnormal conditions in order to help the grid to reliably cope with the contingencies with less impact on frequency. The distribution BESS mitigates the fluctuations caused by renewables and also shifts the load from peak to low load time. These multi-purpose optimizations increase the reliability of the power grid considering the issues of the transmission and distribution networks. The proposed method in this paper solves a few problems of the transmission and distribution networks through calculation of optimal two-purpose BESS.

\section{A. Literature Review}

The majority of published papers in the storage area only focus on operation related to the distribution network. There are less publications about optimal location(s) of the BESS in comparison with optimal sizing due to the complexity of the finding of the optimal locations $[2,3]$. Thus, in many of the previous studies, it was posited that BESS is located near wind farms and large loads. The literature review can be divided into two categories: the first contains storage purposes while the second includes storage sizing and siting.

\section{1) Storage Purposes}

The majority of studies in the operation area are based on uncertainty and fluctuations related to distributed generations. Varkani et al. [4] modeled these uncertainties using Artificial Neural Networks (ANN) and stochastic programming. They discussed joint bidding design by a wind farm and a pumped hydro plant in the day-ahead and ancillary services markets. Forecasting electricity price and wind generation are two good optimization parameters which Gonzalez et al. [5] used in their study for a pumped hydro storage plant. In their study, another approach for joint market participation was proposed by a wind power plant and a pumped hydro storage plant. Pandžić [6] proposed a method based on bilateral contracts and one day-ahead pricing in order to maximize the power plant profit considering the 
112 storage system, photovoltaic system, and a conventional generating unit. This model was for bilateral 113 contracts and a day-ahead market. The conclusions were based on the importance of an accurate evaluation 114 of the storage unit's energy and power ratings. Another stochastic model, based on real-time marketing, 115 was proposed by Pandžić [7] for a similar power plant utilizing wind generation instead of photovoltaic 116 system. Pandžić used a real-time market to balance the day-ahead market bids and the actual generation. 117 Another scheduling method was proposed by Korpaas [8] based on dynamic programing in order to 118 calculate the optimal electricity exchanges considering one transmission line constraints related to wind

119

120

121

122

123

124

125

126

127

128

129

130

131

132

133

134

135

136

137

138

139

140

141

142

143

144

145

146

147

148

149

150

151

152

153

154

155

156

157

158

159

160

161 generation and storage. However, only one transmission line (between the storage and the rest of the transmission network) was considered. Simulation results show that energy storage enables the owners of the wind power plant to take advantage of variations in the spot price, therefore, increasing the value of wind power in the electricity market. An optimal commitment policy considered conversion losses and energy prices in a grid which included wind farm and storage [9]. Zhou et al. [10] modeled a system, based on Markov decision prices and investigated wind farms, storage, and transmission systems. They proved that storage can increase the monetary value of the system. Cahndy et al. [11] performed optimal power flow on a power grid including the storage system and considered the integration of renewable energy resources. Therefore, this study was technical rather than economical. One economical study was done by Faghih et al. [12] with the goal of investigating optimal utilization of storage and the economic value of storage. They considered ramp-rate constraints and different electricity prices and they proved that optimal utilization of storage by consumers can induce price elasticity, particularly around the average price. Nick et al. [13] performed an optimal day ahead scheduling of energy resources in active distribution networks. These resources included dispersed energy storage systems and volatile renewable embedded generators. Nick et al. proposed a convex formulation of optimal power flow to compute the resources schedule. A multi-objective optimization was done by Steta [14] in order to obtain an optimal operation approach for a lossy energy storage system. They used a predictive control model based on a linearized version of an adiabatic compressed air energy storage plant.

\section{2) Storage siting and sizing}

Harsha and Dahleh completed an optimal storage analysis focused on a renewable generator that could support a part of local demand via storing excess generation [15]. Their objective function could find the optimal storage capacity in order to minimize the long-term average cost of demand not served by renewable generation. A similar study was done to find the optimal BESS sizing considering renewable energy resources and dynamic pricing [16]. Formulating the problem as a stochastic dynamic programing model helped the authors to minimize the long-term average cost of conventional generation, as well as the investment in storage, if any, while satisfying the demand. They proved that under fixed electricity prices, storage is profitable if the ratio of the amortized cost of storage to the price of electricity is less than 1/4. A probabilistic reliability analyses was done by Zhang [17] in order to find the optimal size of the storage system that considered the transmission aspects. This study was also technical and did not focus on economical analysis. Dvijotham [18] used an algorithm to find the optimal locations of BESS in the grid. Their method was based on solving an optimization in order to find the minimum number of the optimal places considering the renewable energy forecasts. They used statistics describing usage patterns to reduce the number of storage devices. Denholm and Shioshans completed an analysis for co-locating a wing generation and storage system to decrease transmission costs. They also showed that using the BESS adjacent to wind generations is less attractive than using them near the big loads if the storage device can take advantage of high-value ancillary or capacity services [19].

Arabali et al. [20] performed a cost analysis on a power grid in order to find the optimal placement of BESS using probabilistic optimal power flow. They used Particle Swarm Optimization (PSO) to minimize the sum of operation and congestion costs. A similar optimization method was used by Kerdphol in order to find optimal BESS with load shedding scheme when disconnection of the micro grid from the grid occurs [21]. The results proved that the optimal size of BESS-based PSO with load shedding scheme can achieve higher performance of frequency control compared to the optimal size of BESS-based analytic algorithm 
162

163

164

165

166

167

168

169

170

171

172

173

174

175

176

177

178

\section{9}

180

181

182

183

184

185

186 with load shedding scheme. Li [22] presented a control strategy to improve the smoothing performance in a power system including wind farms, photovoltaic generations, and BESS. This method was based on decreasing the fluctuations of wind and photovoltaic generations and regulating battery state of charge (SOC). Awad [23] proposed a method for the cost-effective improvement of reliability via the allocation of distributed storage units in the distribution systems. The purpose of their study was to optimize the installation cost of BESS with respect to reliability aspects of the grid. Nick et al. [24] proposed a multi objective procedure in order to find optimal siting and sizing of distributed storage system to provide ancillary services. Their objective functions were voltage support and minimizing the network losses along with minimization of the energy cost from grid. In another study, optimal location of distributed storage system was found in [25] to minimize the network voltage deviation. This study was based on formulation of a mixed-integer linear programing problem. An optimal trade-off between technical and economical goals was performed in [26] to find the optimal allocation of dispersed energy storage system in active distribution networks. Their model of dispersed energy storage was able to support the network by both active and reactive power. In [27], a model predictive control strategy is used to smooth out wind power fluctuations thus making the wind power more dispatchable. In this study, the authors showed that the control strategy is important to improve BESS performance for the mentioned application.

\section{B. Contributions of the Heuristic Method}

The proposed methodology in this paper focuses on distribution and transmission issues. This multipurpose algorithm not only covers regular usages of the storage to smooth output power of renewable energy resources in the distribution network, but controls the frequency and provides power to cope with the contingencies in the transmission network. In abnormal situations of the grid, BESS compensates for power shortages in order to regulate the grid frequency. This study contains both technical and economical analysis. Using the proposed method is cost-efficient and the optimal storage can increase the grid reliability.

In this study, the problem is defined in the centralized system and utility operates the BESS along with conventional generators. In terms of the optimal location and capacity of the BESS in the transmission side, a wide variety of contingencies are analyzed in order to find the worst case scenario in a power grid. The applied contingencies are: losing wind or thermal generations and sudden or slow change in wind generations. The transmission BESS decreases the required reserve capacity which is necessary to cope with the contingencies. On the distribution side, BESS is used to smooth the load curve and to remove fluctuations. This will decrease the reserve capacity allocated to deal with abrupt changes in the load. Peak shaving uses excess renewable energy generation in the low load time period and dumps back the stored energy in the peak load time. This helps to shift the renewable energy generation and replace the conventional unit power production. Peak shaving and load smoothing are investigated giving flexibility to the power system operator to manage the available reserve effectively in a timely manner.

Unit commitment problem is solved to find the optimal set of generators to meet the load demand. Moreover, in order to have the maximum benefit in power production under abnormal conditions, an Economic Dispatch (ED) has been performed to decide the optimal amount of power from generators and BESS.

\section{FORMULATION AND DEFINITIONS}

The following subsections contain details about the tools used in the transmission part of BESS including: TDPF, CVNN, and ED.

\section{1) TDPF Formulation}

The power flow problem can mathematically be defined as: 
$\left[I_{\text {Bus }}\right]=\left[Y_{\text {Bus }}\right] \cdot\left[V_{\text {Bus }}\right]$

210 The active and reactive power flows $(\Delta P, \Delta Q)$ can be calculated based on the change of voltage amplitudes 211 and angles $(\Delta V, \Delta \theta)$, which equation (2) shows that:

212

$\left[\begin{array}{c}\Delta P \\ \Delta Q\end{array}\right]=\left[\begin{array}{cc}\frac{\partial P}{\partial \theta} & 0 \\ 0 & \frac{\partial Q}{\partial V} V\end{array}\right]\left[\begin{array}{l}\Delta \theta \\ \frac{\Delta V}{V}\end{array}\right]$

213 Where the differential matrix is Jacobian. Newton-Raphson algorithm has been used to solve the power 214 flow equations [28].

215

216

217

218

TDPF performs power flow during a time interval. In TDPF simulations, the grid's equipments have been modeled dynamically not statically. Time domain simulation includes solving the following problems for a typical time $t$ and time step $\Delta t$ :

$f(x(t+\Delta t), y(t+\Delta t), f(t))=0$

$g(x(t+\Delta t), y(t+\Delta t))=0$

219

220

221

222

223

Equations (3) and (4) are nonlinear and have been solved using Newton-Raphson method. This method repeatedly calculates the increments $\Delta x^{i}$ and $\Delta y^{i}$ of the state and algebraic variables and then updates the actual variables:

$$
\begin{aligned}
& {\left[\begin{array}{c}
\Delta x^{i} \\
\Delta y^{i}
\end{array}\right]=-\left[A_{c}^{i}\right]^{-1}\left[\begin{array}{c}
f_{n}^{i} \\
g^{i}
\end{array}\right]} \\
& x^{i+1}=x^{i}+\Delta x^{i} \\
& y^{i+1}=y^{i}+\Delta y^{i}
\end{aligned}
$$

The loop stops when the variable increment is less than a certain fixed tolerance or when the iteration number reaches its maximum value. There are two techniques to solve the TDPF equations: Forward Euler Method and Trapezoidal Method. The first technique has been used in this study [29] [30].

By modeling the grid with TDPF, dynamic behaviors of the grid are analyzed and eigenvalues of dynamic system can be moved to ensure the grid stability. Additionally, the contingencies or disturbances can be easily applied to the model in order to analyze their effects on the grid. Therefore, appropriate strategies can be adopted to have the most optimized reaction to overcome the contingency or fault. In this study TDPF, is performed using PSAT toolbox in MATLAB [29].

\section{2) CVNN Formulation}

ANNs are widely used in energy systems applications. They can learn from the examples and perform forecasts in high speeds [31] [32]. The major difference between ANN and CVNN is the nature of using parameters. A real number is a subset of complex number, thus CVNN is an extension of ANN. CVNN is the complex form of ANN which is appropriate for the systems with the complex parameters such as power grid [33]. In this study, CVNN uses the load data of the grid in order to forecast the parameters in times ahead (either normal or atypical conditions ahead) [34]. Since, time plays a critical role in contingency management, it is more desirable to have a smaller system which produces the required results without 
dealing with computation burden. CVNN learns the power grid for different contingencies offline and creates a system which can easily and quickly find the contingency parameters.

In this paper, a multi-stage learning process has been used by CVNN to forecast the voltage amplitudes and angles under contingencies. Equation (2) has been used for two stages of learning: one stage is learning through the active powers and voltage angles and another stage is learning through the reactive powers and voltage amplitudes. Fig. 1 shows two stages of learning by CVNN. The parameters at the top and bottom of the arrows are related to the first and second learning stages, respectively. The input vectors of CVNN block (in time $t$ ) include two sections: the first input vector is the voltage amplitudes of last $N L$ time series (including: $\left.V_{t-1}, V_{t-2}, \ldots, V_{t-N L}\right)$ and previous reactive power $\left(Q_{t-1}\right)$, and the second input-vector is the voltage angles of last $N L$ time series (including: $\left.\theta_{t-1}, \theta_{t-2}, \ldots, \theta_{t-N L}\right)$ and previous active power $\left(P_{t-1}\right)$. The outputs of CVNN block are forecast values of the voltage amplitudes and angles in time $t\left(V_{t}, \theta_{t}\right)$. NL is the minimum necessary number of previous parameters to learn the system well. All of the above parameters are defined for the whole grid buses.

Block diagram of Fig. 2 indicates the complete steps of forecasting process using the proposed method which is a combination of CVNN and TDPF. The role of TDPF is to create the previous values of the voltage amplitudes and angles using the active and reactive powers of the grid. CVNN uses the previous values to learn the system and to forecast the voltage amplitudes and angles in time(s) ahead.

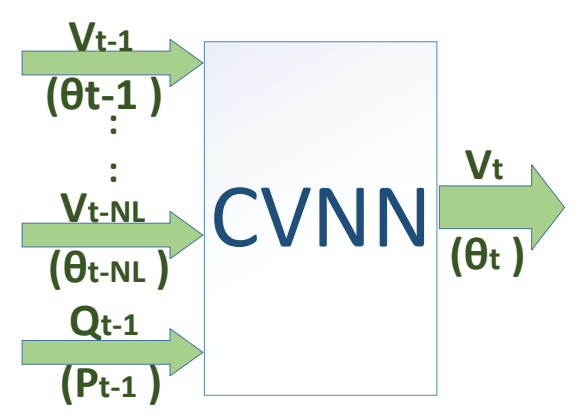

Figure 1. Two stages of learning by CVNN to forecast the voltage amplitudes and angles

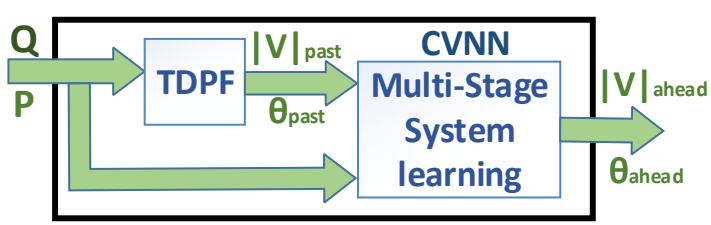

Figure 2. Combination of TDPF and CVNN to forecast the time-ahead parameters

\section{3) ED Formulation}

One of the purposes of combining the two previous tools (TDPF and CVNN) is to determine the optimal location(s) of BESS. These locations are sensitive buses of the grid. The other purpose is to calculate the power needed in order to cope with the contingencies and regulate the grid frequency. Next, it is necessary to calculate the optimal output of the generators and BESS to produce the required power economically. ED is the best tool to calculate how much power should be generated by each power source. For the proposed methodology, the generators of the grid are allocated into two groups: local generators (located in the sensitive buses) and distant generators (connected to the sensitive buses with transmission lines). The distant generators located far away from the sensitive buses. The objective function for the ED is a cost function with the following terms: 
$=\sum_{i=1}^{N G \text { local }} C_{G E N, i}^{\text {local }}+\sum_{j=1}^{N G \text { dist }} C_{G E N, j}^{\text {dist }}+\sum_{k=1}^{N B E S S} C_{B E S S, k}^{\text {total }}$

281 Where;

$$
\begin{aligned}
C_{G E N, i}^{\text {local }}= & a_{\text {local }, i}+b_{\text {local }, i} \cdot P_{G N,, i}^{\text {local }}+c_{\text {local }, i} \cdot\left(P_{G E N, i}^{\text {local }}\right)^{2} \\
C_{G E N, j}^{\text {dist },}= & a_{\text {dist }, j}+b_{\text {dist }, j} \cdot P_{G E N, j}^{\text {dist }}+c_{\text {dist }, j} \cdot\left(P_{G E N, j}^{\text {dist }, j}\right)^{2} \\
& +\sum_{l=1}^{N E E S S} C_{j, l}^{\text {trans }}
\end{aligned}
$$

$C_{B E S S, k}^{\text {total }}=C_{B E S S, k}^{s t}+C_{B E S S, k}^{p c s}$

$282 C_{j, l}^{\text {trans }}$ is a quadratic function of transmitted power:

Transmitted power $=\sum_{l=1}^{N B E S S} R_{j, l}+j X_{j, l} \mid \cdot I_{j, l}{ }^{2}$

$=\sum_{l=1}^{N B E S S}\left|R_{j, l}+j X_{j, l}\right| \cdot\left(\frac{P_{j, l}^{\text {flow }}}{V_{j, l}}\right)^{2}$

283 The components of the total cost of BESS are:

$$
\begin{aligned}
& C_{B E S S, k}^{s t}=C_{B E S S, k}^{s t, u n i t} \cdot P_{B E S S, k} \cdot\left(\frac{1}{\eta}\right) \\
& C_{B E S S, k}^{p c s}=C_{B E S S, k}^{p c s, u n i t} \cdot P_{B E S S, k}
\end{aligned}
$$

Where $\eta$ is the inefficiency factor of the storage device [35]. The constraints of the optimization are:

$$
\begin{aligned}
& P_{G E N \text { min }, i}^{\text {local }} \leq P_{G E N, i}^{\text {local }} \leq P_{G E N \max , i}^{\text {local }} \\
& P_{G E N \text { min }, j}^{\text {dist }} \leq P_{G E N, j}^{\text {dist }} \leq P_{G E N \max , j}^{\text {dist }} \\
& \sum_{i=1}^{N G \text { local }} P_{G E N, i}^{\text {local }}+\sum_{j=1}^{N G \text { dist }} P_{G E N, j}^{\text {dist }}+\sum_{k=1}^{N B E S S} P_{B E S S, k}=\sum_{m=1}^{N B E S S} P_{\text {req }, m}
\end{aligned}
$$

The proposed method in this paper includes economical and technical optimization of the capacity and location(s) of BESS in the power grid considering the issues of transmission and distribution networks. Fig. 3 shows the whole steps of the methodology in two parts. The sections below, A and B, describe the proposed approach for the transmission and distribution parts, respectively.

\section{A. Transmission Part}

The purpose of the transmission part (upper part of Fig. 3) is to show how to determine the optimal place(s) and capacity of BESS to control the frequency and find the best reaction in the abnormal situations 
to successfully and reliably cope with the contingencies. The optimal results of the site(s) and size of the BESS minimizes the cost and power interruption. In this part, three powerful tools have been used: CVNN, $\mathrm{TDPF}$, and ED which were described further in detail in section II.
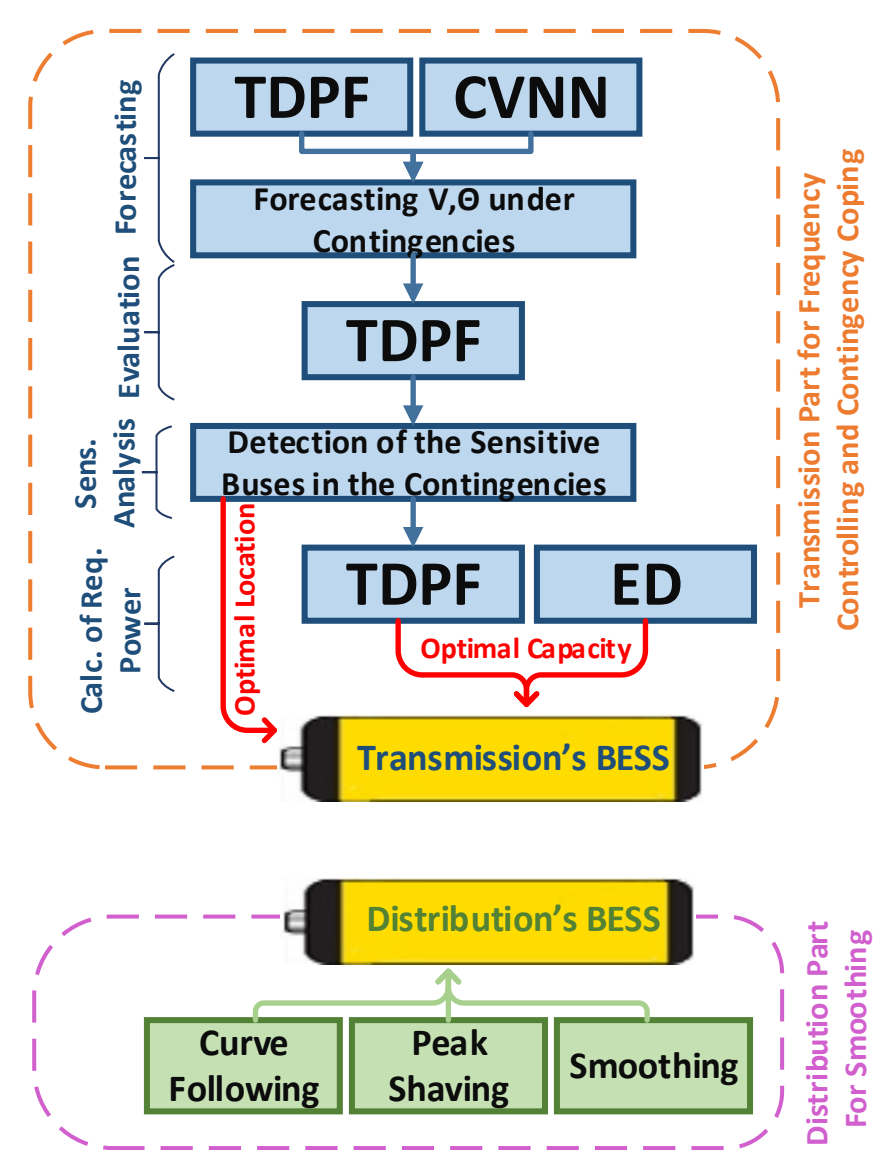

Figure 3. Algorithm of the proposed method including two parts

In the first step of the transmission part (forecasting step in Fig. 3), a heuristic method is applied to the load data of the grid to forecast the voltage amplitudes and angles under different contingencies. This method is a combination of CVNN and TDPF. The second step is to compare the forecast values with actual data to ensure accuracy of forecasting (evaluation step in Fig. 3). Next, a sensitive analysis is performed to detect the sensitive buses of the grid under a wide range of contingencies based on forecast results (sens. analysis step in Fig. 3). Obviously, voltage amplitudes and angles of the buses change during the contingencies which lead to changes in the grid frequency. Therefore, the variations of the voltage amplitudes and angles are suitable indices in detecting the sensitive buses. Under contingencies, these buses have the highest variations in the mentioned parameters compared to other buses. Thus, adding the storage in these sensitive buses can optimally regulate the grid frequency. The optimal location(s) of the BESS are these sensitive busses which consider the effects of the whole range of the contingencies (including the worst case scenario). In this study the applied contingencies are: losing wind or thermal generations and sudden or gradual change in wind generations. Generally, some of the sensitive buses are positioned close to the wind generations. In the last step of the transmission part, the required power is calculated using TDPF. This power is the necessary active power which should be injected to the grid through the sensitive buses in order to control the frequency and compensate for power shortages in the worst case contingency. Running an ED determines how much power should be produced by each generator and BESS to have the minimum cost (calc. of req. power step in Fig. 3). Combination of TDPF 
and ED calculates the optimal contribution of generators and BESS for power compensation in the transmission part.

\section{B. Distribution Part}

The purposes of the distribution BESS (lower part of Fig. 3) are peak shaving and load curve smoothing. The following subsections provide more details.

\section{1) Peak shaving}

One of the important services in the distribution grid is peak load shaving [36]. Moving the required energy from the peaks of the load curve into the valleys assists the utility to reduce committed units and efficiently utilize renewable energy resources by using energy already stored in BESS to meet the demand. The underlying concept for peak load shaving is in the form of a quadratic programming method which flattens the load curve:

$$
\sum_{t=1}^{N}\left(P_{b a t}(t)+P_{c i r}(t)\right)^{2}
$$

Subject to BESS capacity constraints:

$\frac{1}{\Delta t}\left[S O C_{\min } E_{t o t}-E(t)\right]<P_{b a t}(t)$

$\frac{1}{\Delta t}\left[S O C_{\max } E_{t o t}-E(t)\right]>P_{b a t}(t)$

According to the following equation, energy of BESS is related to SOC:

$E(t+1)=E(t)+P_{b a t}(t) \Delta t$

We can also force BESS to follow a reference load curve,

Minimize $\sum_{t=1}^{N}\left(P_{\text {bat }}(t)+P_{\text {cir }}(t)-P_{\text {ref }}(t)\right)^{2}$

\section{2) Load Curve Smoothing}

With growing penetration of distributed renewable generations such as rooftop photovoltaic resources, the variability in the aggregate load curve increases accordingly [37]. Additional reserve must be up and running to cope with the abrupt fluctuations in the load curve. BESS can mitigate the sharp variations in the power curve to save some time for rapid startup generators to pick up the load [38]. Smoothing out the load curve should keep the SOC constant to save energy for peak shaving purpose. BESS will remove fluctuations around a straight line rather than a horizontal line. The slope of this line is affected by the load forecast, SOC deviation from the desired point, and the startup time of available generators in the grid. The line connects the current load point and 20 minutes ahead forecast load point:

$P_{L}(t)=\left(P_{b a t}\left(t_{k}\right)+P_{c i r}\left(t_{k}\right)\right)+m\left(t-t_{k}\right)$

Where the slope $(m)$ is given by:

$m=\frac{P_{c i r}\left(t_{k+1}\right)-\left(P_{b a t}\left(t_{k}\right)+P_{c i r}\left(t_{k}\right)\right)+g\left(\operatorname{SOC}\left(t_{k}\right)\right)}{t_{k+1}-t_{k}}$ 
The last term in the slope compensates the SOC deviation from the desired point which is usually kept at the highest allowable limit kept for peak shaving service.

\section{CAse Study}

The proposed approach has been tested on a real world model of an island in the United States (island of Maui located in the state of Hawai'i). The island's grid includes 184 buses, 20 generators and two wind farms. Table I provides the generators' data, including coefficients of cost functions and generation constraints. These generators have the available capacity to produce power in the contingencies. This case study has been modeled using the real data of the grid's equipment, loads, and generators. In the Maui grid, the wind generations have the highest priority to inject the power, according to the contract with the electric utility. This study has been modeled for simulation during times of peak-load with a reserve of $10 \%$ total capacity. In order to forecast the voltage amplitudes and angles in the abnormal conditions, different contingencies were applied to the grid model including: gradual and sudden change in wind generations and losing thermal generators. In this study, a wide range of scenarios have been considered in order to determine the worst case contingency of the grid.

Table I. Cost coefficients and constraints of the available generators in the abnormal situation in Maui grid (with fuel cost of \$12/MBtu) [39]

\begin{tabular}{|c|c|c|c|c|c|}
\hline \multicolumn{3}{|c|}{ Cost $=a+b^{*} P+c^{*} P^{\wedge} 2$} & \multicolumn{2}{c|}{$P \min <P<P \max$} \\
\hline \multirow{2}{*}{$\begin{array}{c}\text { Bus } \\
\text { No. }\end{array}$} & $\mathbf{a}$ & $\mathbf{b}$ & $\mathbf{c}$ & \multicolumn{2}{c|}{$\begin{array}{c}\text { Power } \\
\text { Constraints }\end{array}$} \\
\cline { 5 - 7 } & & & & $\begin{array}{c}\text { Pmin } \\
(\mathbf{M W})\end{array}$ & $\begin{array}{c}\text { Pmax } \\
(\mathbf{M W})\end{array}$ \\
\hline 48 & 18.997 & 1.180 & 1.002 & 0 & 1.8 \\
\hline 49 & 8.868 & 12.231 & 0.282 & 0 & 2.2 \\
\hline 50 & 7.931 & 11.926 & 0.411 & 0 & 3.3 \\
\hline 53 & 25.624 & -2.922 & 1.477 & 0 & 2.8 \\
\hline 140 & 14.680 & 6.588 & 0.052 & 0 & 2.2 \\
\hline 181 & 24.598 & 3.762 & 0.289 & 0 & 2.5 \\
\hline
\end{tabular}

\section{RESULTS}

This section contains the results of transmission and distribution parts in order to find the optimal locations and capacity of the storage system.

\section{A. Transmission Part Results}

After ensuring the accuracy of the forecast parameters, a sensitive analysis is carried out (according to sen. analysis step in Fig. 3). In this step, a wide range of contingencies have been applied to the grid and the impact of all of them are considered to find the optimal location(s) of the BESS. Next, for finding the optimal capacity of BESS, the worst case contingency is considered. Fig. 4 shows load curve (MW) of Maui grid in one day. Fig. 5 demonstrates the first set of scenarios, which is sudden change of both wind generations from their maximum output to zero in a short time (10 minutes). The second set of scenarios is change the wind generations, based on meteorological data of Maui, which has been shown in Fig. 6. This figure demonstrates changes of the wind generations in one hour of day which sometimes the wind generations decrease significantly. Losing the thermal generations in different times of day is the third set of scenarios, which Fig. 7 represents it. Each color demonstrates one generator. The dashed red line represents the worst case contingency (losing the largest generation). 
411

412

413

414

415

416

417

418

419

420

421

422

423

424

425

426

427

428

429

430

431

432

433

434

435

436

437

438

439

440

441

442

443

444

445

446

447

448

449

450

451

452

453

454

455

456

457

458

459

460

461

462

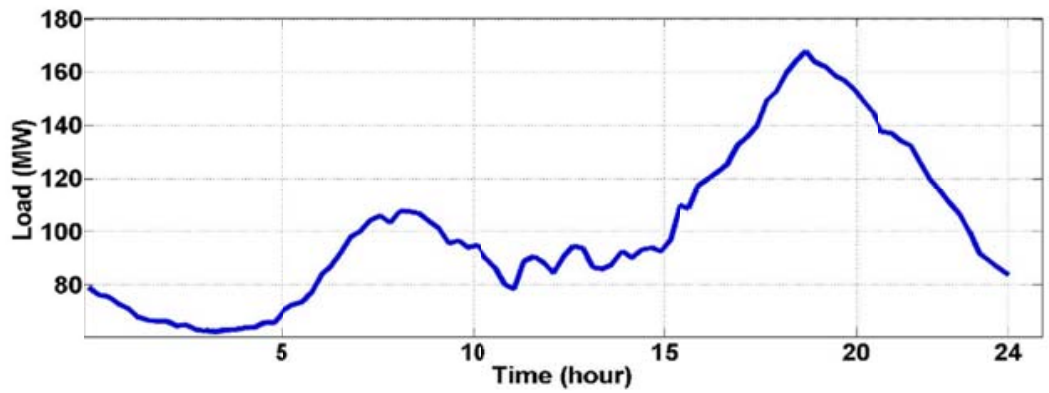

Figure 4. Load (MW) in one day of Maui [39]

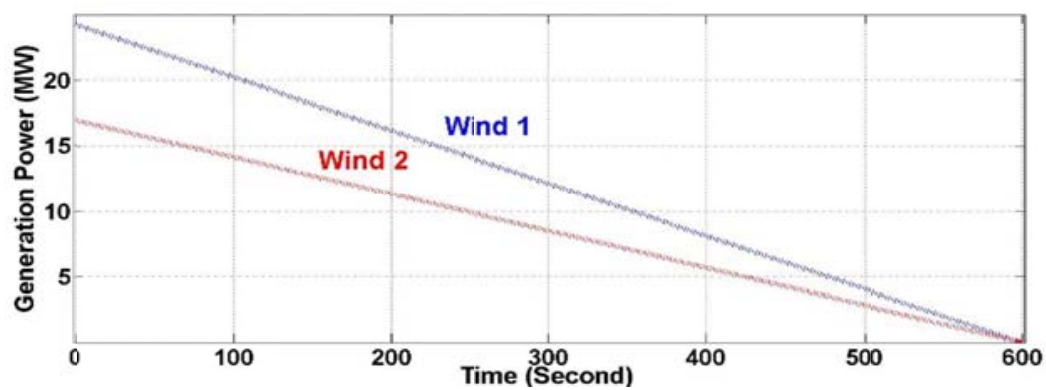

Figure 5. The first set of scenarios - Decreasing the wind outputs from maximum to zero

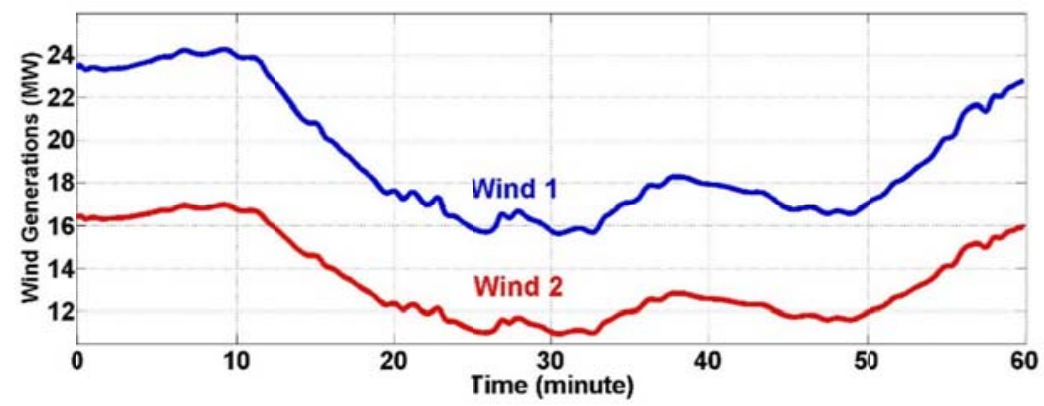

Figure 6. The second set of scenarios - Changing the wind generations in one hour of day [39]

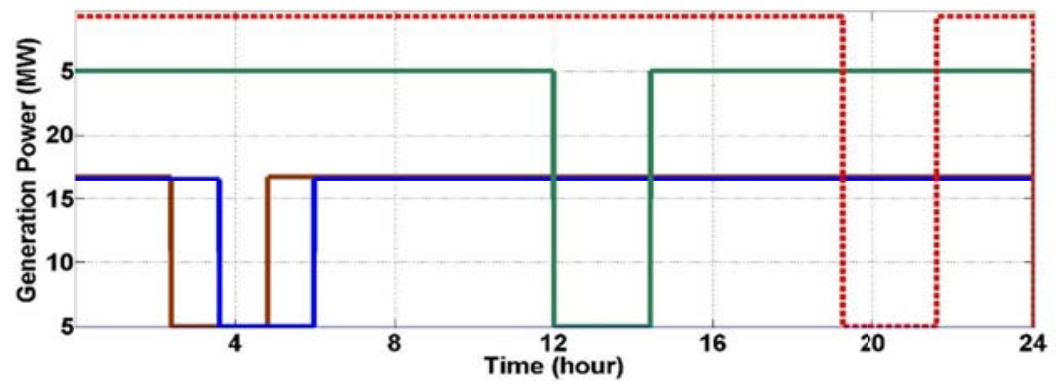

Figure 7. The third set of scenarios - losing the thermal generations in different times of day (including the worst case contingency) 
463

464

465

466

467

468

469

470

471

472

473

474

475

476

477

478

479

480

481

482

483

484

485

486

487

488

489

490

491

492

493

494

495

496

497

498

499

500

501

502

503

504

505

506

507

508

509

510

511

512

513

514

515

Fig. 8 shows the results of the sensitivity analysis during a wide range of contingencies to detect the sensitive buses as the optimal locations of the BESS. The sensitivity analysis shows bus numbers 166 and 180 have the maximum sensitivity in different contingencies. Therefore, these buses are the best places of the BESS (optimal locations of the BESS). Bus 180 is a sensitive generation bus which is appropriate for the transmission BESS and bus 166 is a sensitive load bus which is appropriate for the distribution BESS. Using the storage system in these two buses not only regulates the frequency in the abnormal conditions but also compensates for the power shortage in the contingencies to have the minimum power interruption.
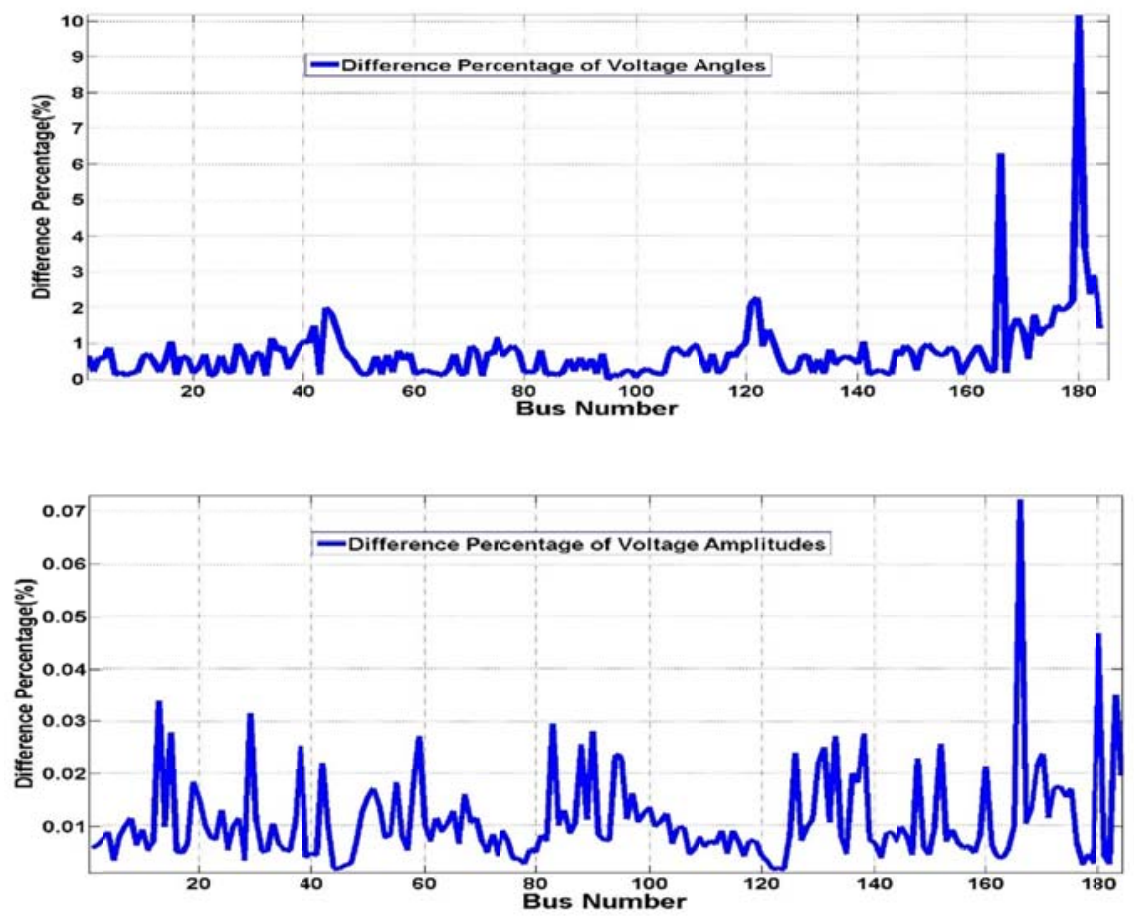

Figure 8. Results of the sensitivity analysis for the voltage amplitudes and angles in a wide range of contingencies

After finding the optimal locations of the BESS, the capacity of the transmission and distribution BESS should be calculated. In order to calculate the required active power in the transmission part (according to calc. of req. power step in Fig. 3), the worst case scenario of contingency has been considered which is losing the biggest generation with $24.3 \mathrm{MW}$ production. With running TDPF under the worst case contingency and using the equation (2), the required power can be calculated. This power should be injected (through the sensitive buses) into the grid to cope with the abnormal situations and regulate the frequency. In the worst case scenario of Maui grid, $15.73 \mathrm{MW}$ power should be injected from through the sensitive buses to compensate for the power shortage.

Table II shows data of transmission lines which connect the available generators of Maui to two sensitive buses. The transmission line impedance is $\mathrm{Z}=0.05+0.47 \mathrm{j} \Omega$ /mile. Table III illustrates the results of ED for the both sensitive buses. The optimal output of power sources (including generators and BESS) have been calculated, at the lowest possible cost, subject to transmission and operational constraints.

Table II. Transmission lines information for connecting the generators to two sensitive buses

\begin{tabular}{|c|c|c|}
\hline \multirow{2}{*}{$\begin{array}{c}\text { From Bus } \\
\text { No. }\end{array}$} & \multicolumn{2}{|c|}{ Transmission line distance (mile) } \\
\cline { 2 - 3 } & $\begin{array}{c}\text { To the first } \\
\text { sensitive bus } \\
(166)\end{array}$ & $\begin{array}{c}\text { To the second } \\
\text { sensitive bus } \\
(180)\end{array}$ \\
\hline 48 & 15 & 4.5 \\
\hline 49 & 30 & 7.5 \\
\hline 50 & 40 & 8 \\
\hline 53 & 80 & 75 \\
\hline 140 & 60 & 13 \\
\hline 181 & 10 & 29 \\
\hline
\end{tabular}


Table III. ED results: optimal outputs of the available generators and BESS to cope with the worst case of contingency and regulate the frequency (in transmission part of BESS)

\begin{tabular}{|c|c|}
\hline Power Source & $\begin{array}{c}\text { Generation Power }(\mathrm{MW}) \\
\text { (based on ED results) }\end{array}$ \\
\hline Gen. bus \#48 & 1.8 \\
\hline Gen. bus \#49 & 2.2 \\
\hline Gen. bus \#50 & 3.3 \\
\hline Gen. bus \#53 & 2.8 \\
\hline Gen. bus \#140 & 2.2 \\
\hline Gen. bus \#181 & 2.5 \\
\hline BESS bus\#180 & $\mathbf{0 . 9 3}$ \\
\hline
\end{tabular}

The transmission BESS (for contingency management and frequency control) should be allocated in bus 180 (sensitive generation bus). Based on the results in table III, the required power for the BESS is 0.93 MW. In order to have the maximum life and efficiency of the BESS, it is recommended to use the BESS in the range between $S O C_{\min }$ and $S O C_{\max }$. For the given values of 0.2 and 0.8 for these parameters, respectively, the active power of the transmission part of the BESS will be:

$0.93 M W^{*} \frac{1}{(0.8-0.2)}=1.55 M W$

Historical data for Maui island shows that the maximum time for the contingency (especially losing the generation) was one hour. Thus, the energy of 1.55MWhr should be supplied by BESS to cope with the contingencies and regulate the frequency.

\section{B. Distribution Part Results}

The proposed method for the distribution part includes two techniques: peak shaving and load curve smoothing. In this section, the results of applying peak shaving technique are presented in Fig. 9 and 10. Next, the results of applying both techniques simultaneously are presented Fig. 11 and 12 .

Peak shaving is implemented on an actual distribution load curve shown in Fig. 9. Since there is less uncertainty in forecasting the load in the early morning and night, peak shaving performs well during time periods. A better load forecast around noon when photovoltaic generation comes online, will help to reach a flatter load curve.

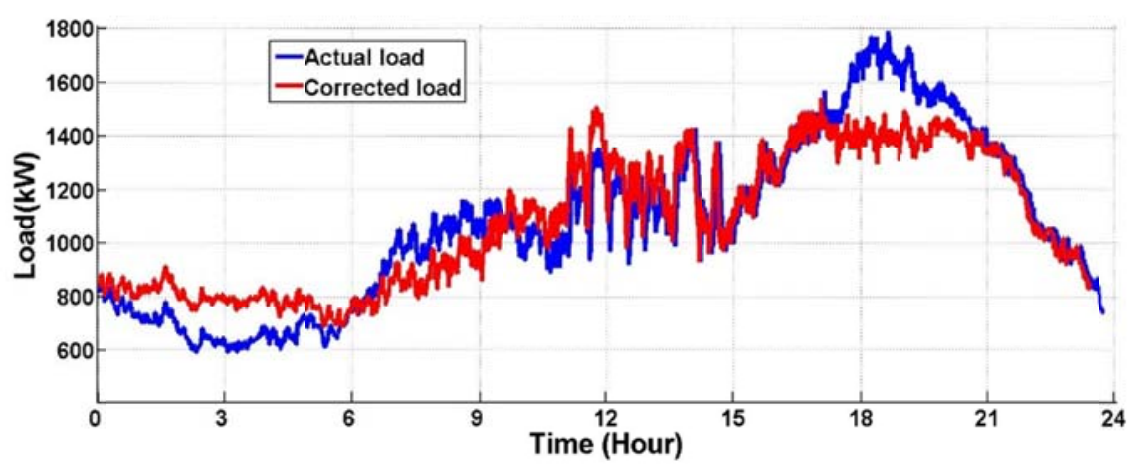

Figure 9. Peak shaving for the distribution grid

The SOC graph for the corresponding peak shaving purpose is shown in Fig. 10. The higher and lower SOC limits are assumed to be 0.8 and 0.2 respectively to increase the BESS lifetime. The SOC curve can be obtained from a reference power curve obtained from a combination of power system operation study and renewable generation forecast. Load smoothing is performed on the same distribution load grid and the obtained results are depicted in Fig. 11. The original load has more fluctuation than the corrected load which needs higher spinning reserve to respond effectively to the imbalance of load and generation. 
Moreover, the corrected load is flatter than the original load which decreases the amount of generation form conventional generates in the peak time. As a results, the cost of generation reduces and less fuels is burnt to meet the demand. The corresponding SOC change in Fig. 12 shows that $S O C$ does not alter significantly during smoothing period which occurs around noon. Peak shaving is also implemented in this figure with slight changes in $g(S O C)$. The advantage of this method over the previous one is that it benefits from the real time load information which make the forecast more accurate and SOC planning more reliable.

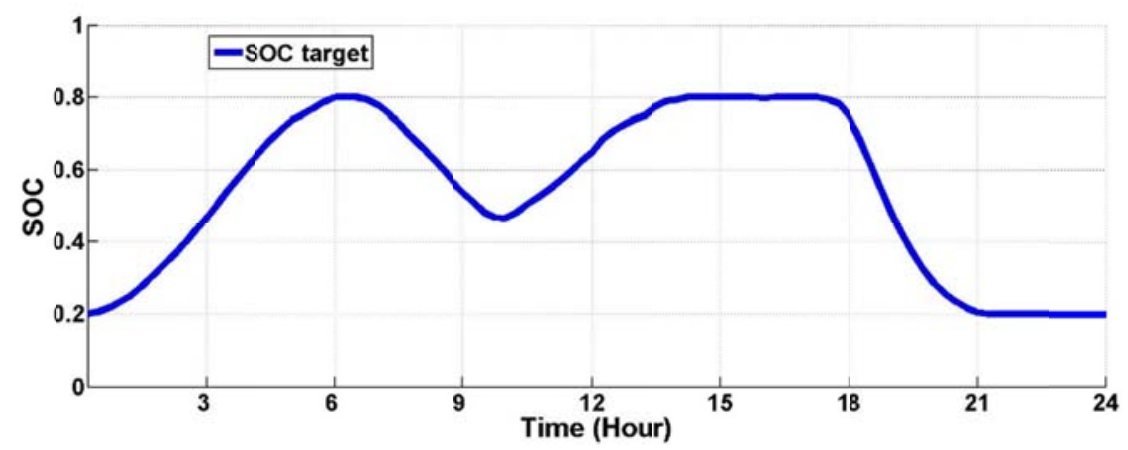

Figure 10. Target SOC obtained for peak shaving purpose

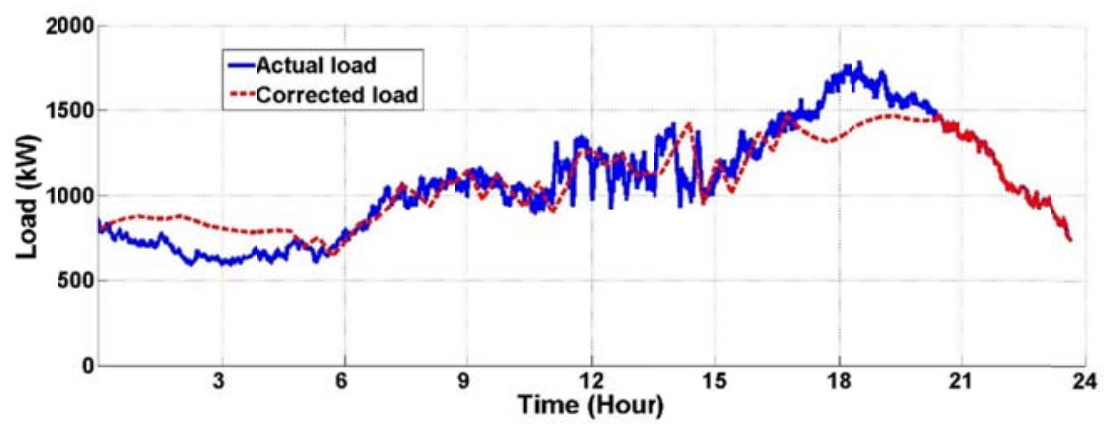

Figure 11. Simultaneous peak shaving and load smoothing for distribution grid with high intermittency

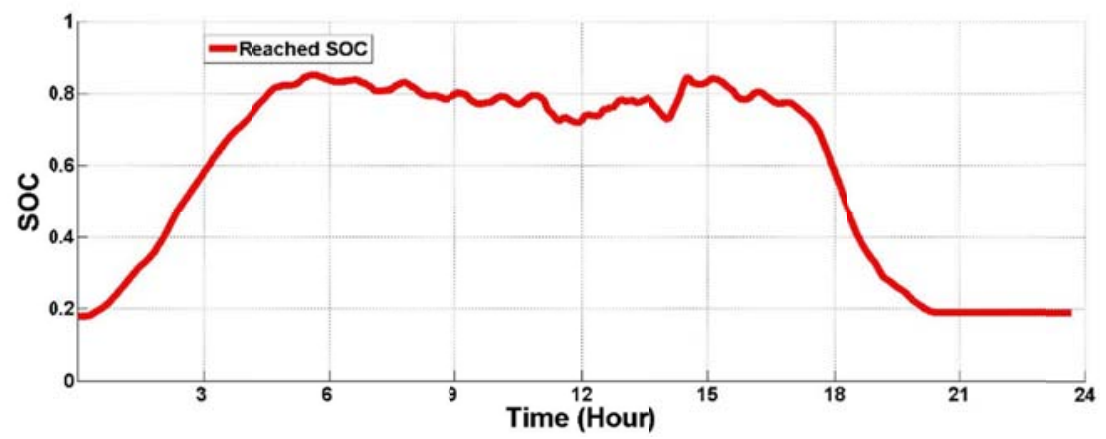

Figure 12. SOC variation for simultaneous peak shaving and load curve smoothing

Based on Fig. 11, calculation the integral of difference between the actual and corrected load in the peak load times leads to the required energy supplied by BESS. This integral will be about $700 \mathrm{kWh}$ from 5-9 
604

605

606

607

608

609

610

611

612

613

614

615

616

617

618

619

620

621

622

623

624

625

626

627

628

629

630

631

632

633

634

635

636

637

638

639

640

641

642

643

644

645

646

647 pm. In order to have the maximum life of the battery, it is better to utilize the BESS in the range between $S O C_{\min }(0.2)$ and $S O C_{\max }(0.8)$. Thus, the capacity of the distribution part of BESS will be:

$0.7 M W h r * \frac{1}{(0.8-0.2)}=1.17 M W h r$

Finally, for the Maui grid, the final results for the capacity of the multi-purpose BESS are: $1.55 \mathrm{MWh}$ for the transmission BESS (located in bus 180 as a generation bus in transmission system) and $1.17 \mathrm{MWh}$ for the distribution BESS (located in bus 166 as a load bus in distribution system). The ratio of the transmission BESS to distribution is 1.32. By allocating the calculated capacities of BESS in the obtained optimal locations of the grid, contingency management and frequency regulation goals can be reached on the transmission side. Moreover, peak shaving and load curve smoothing can be implemented on the distribution side. Fig. 13 shows the difference percentages of the voltage amplitudes and angles before contingency (normal conditions) and during contingency (using optimal BESS). This figure demonstrates the effectiveness of the proposed method to find the optimal locations and capacities of BESS to deal with the worst case contingency.
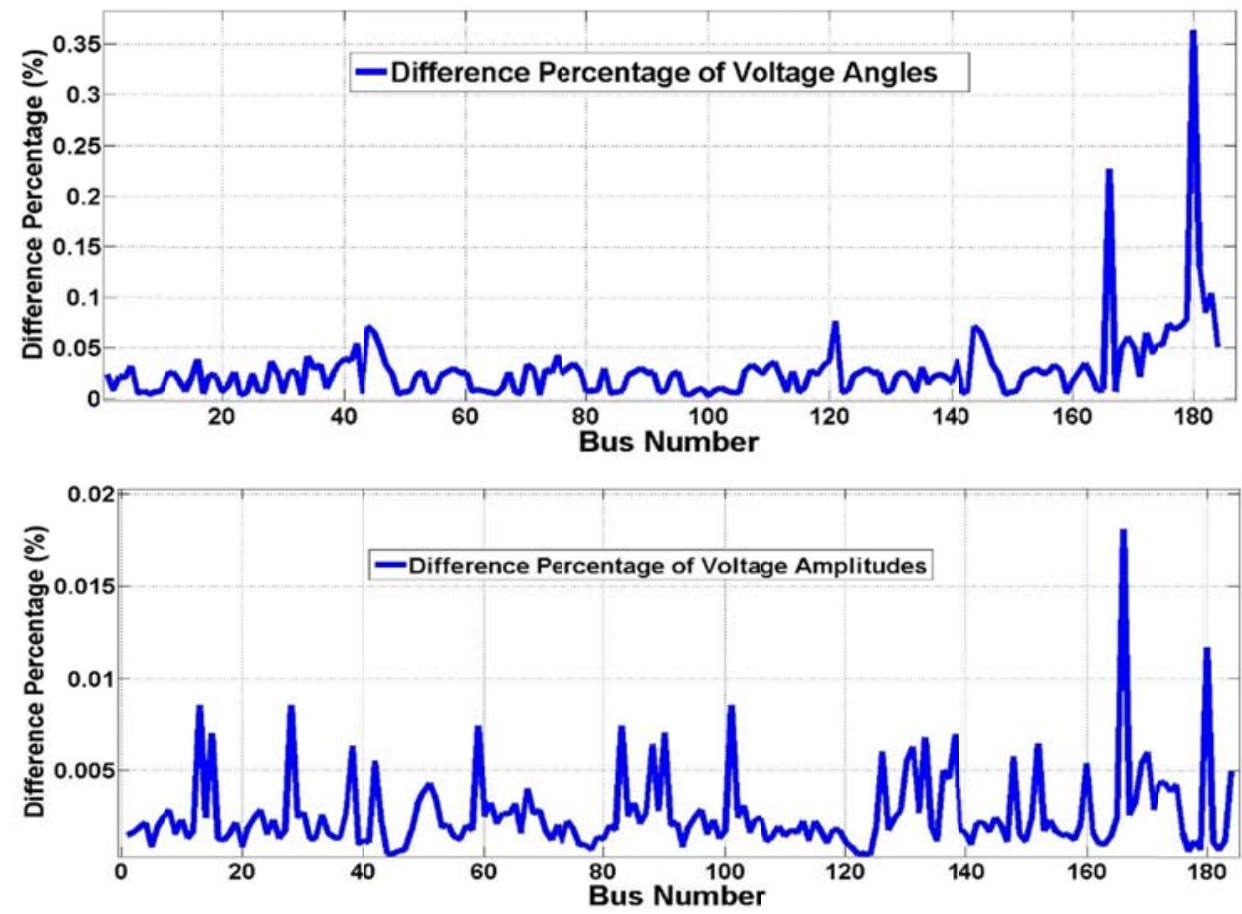

Figure 13. Difference percentages of the voltage amplitudes and angles before contingency (normal conditions) and during contingency (using optimal BESS)

\section{CONCLUSiOnS}

This study proposes a heuristic method in order to find the optimal location(s) and capacity of a multipurpose BESS in the power grid. One objective of the use of the BESS is related to the transmission side of the grid to cope with the contingencies and regulate the grid frequency under abnormal conditions. In this objective, CVNN, TDPF, and ED programs are utilized to perform the optimizations. In the next objective, the BESS is used to manage the distribution system of the grid for peak shaving and load curve smoothing. The proposed heuristic method is applied on a real grid model to find the best location(s) and capacity of the BESS. 
649 This project is sponsored by US National Science Foundation under award number: 1310709.

[1] A. A. Akhil, G. Huff, A. B. Currier, B. C. Kaun, and D. M. Rastler, "DOE/EPRI 2013 Electricity Storage Handbook in Collaboration With NRECA," Sandia National Lab., Rep.SAND2013-513, Jul. 2013.

[2] S. V. Papaefthymiou, S. A. Papathanassiou, "Optimum sizing of wind-pumped-storage hybrid power stations in island systems," Renewable Energy, vol. 64, pp. 187-196, 2014.

[3] J. P. Fossati, A. Galarza, A. Martin-Villate, L. Fontan, "A method for optimal sizing energy storage systems for microgrids," Renewable Energy, vol. 77, pp. 539-549, 2015.

[4] A. K. Varkani, A. Daraeepour,and H. Monsef, "Anewself-scheduling strategy for integrated operation of wind and pumpedstorage power plantsinpowermarkets," Appl.Energy, vol. 88, no. 12, p. 5002-5012, Dec. 2011.

[5] J. Garcia-Gonzalez, R. M. R. delaMuela, L. M. Santos, and A. M. Gonzalez, "Stochastic joint optimization of wind generation and pumpedstorage units in an electricity market," IEEE Trans. Power Syst., vol. 23, no. 2, p. 460-468, May 2008.

[6] H. Pandžić, I. Kuzle, and T. Capuder, "Virtual power plant mid-term dispatchoptimization," Appl. Energy, vol. 101, no. 1, p. 134-141, Jan. 2013.

[7] H. Pandžić, J. M. Morales, A. J. Conejo, and I. Kuzle, "Offering model for a virtual power plant based on stochastic programming," Appl. Energy, vol. 105, no. 5, p. 282-292, May 2013.

[8] M. Korpaas, A. T. Holen, and R. Hildrum, "Operation and sizing of energy storage for wind power plants in a market system," Elect. Power Energy Syst., vol. 25, no. 8, p. 599-606, Oct. 2003.

[9] J. H. Kim and W. B. Powell, "Optimal energy commitments with storage and intermittent supply," Oper. Res., vol. 59, no. 6, p. 1347-1360, Dec. 2011.

[10] Y. Zhou, A. Scheller-Wolf, N. Secomandi, and S. Smith, "Managing Wind-based Electricity Generation in the Presence of Storage and Transmission Capacity," Tepper School of Business, 2013. [Online]. Available: repository.cmu.edu/cgi/viewcontent.cgi?article $=2469 \&$ context=tepper.

[11] K. M. Chandy, S. H. Low, U. Topcu, and H. Xu, "A simple optimal power flow model with energy storage," in 49th IEEE Conf. Decision and Control, Atlanta, GA, USA, Dec. 2010.

[12] A. Faghih, M. Roozbehani, and M. Dahleh, "Optimal utilization of storage and the induced price elasticity of demand in the presence of rampconstraints," in 50th IEEE Conf. Decision and Control and Eur. Control Conf., Orlando, FL, USA, Dec. 2011.

[13] M. Nick, R. Cherkaoui, and M. Paolone, "Stochastic Day-ahead Optimal Scheduling of Active Distribution Networks with Dispersed Energy Storage and Renewable Resources," in IEEE Conference on Technologies for Sustainability (SusTech), 2014.

[14] F. De Samaniego Steta, A. Ulbig, S. Koch, and G. Andersson, "A model-based optimal operation strategy for lossy energy storage systems: The case of compressed air energy storage plants," in Power System Computation Conf., Stockholm, Sweden, Aug.2011.

[15] P. Harsha and M. Dahleh, "Optimal sizing of energy storage for efficient integration of renewable energy," in 50th IEEE Conf. Decision and Control and Eur. Control Conf., Orlando, FL, USA, Dec. 2011.

[16] P. Harsha and M. Dahleh, "Optimal Management and Sizing of Energy Storage Under Dynamic Pricing for the Efficient Integration of Renewable Energy," IEEE Trans. Power Syst., vol. 30, no. 3, pp. 1164-1181, 2012.

[17] Y. Zhang, S. Zhu, and A. A. Chowdhury, "Reliability Modeling and Control Schemes of Composite Energy Storage and Wind Generation System With Adequate Transmission Upgrades," IEEE Trans. Sustain. Energy, vol. 2, no. 4, p. 520-526, Oct. 2011.

[18] K. Dvijotham, S. Backhaus, and M. Chertkov, "Operations-Based Planning for Placement and Sizing of Energy Storage in a Grid With a High Penetration of Renewables," arXiv:1107.1382v2, Jul. 2011.

[19] P. Denholm and R. Sioshansi, "The value of compressed air energy storage with wind in transmission-constrained electric power systems," Energy Policy, vol. 37, no. 8, p. 3149-3158, Aug. 2009.

[20] A. Arabali, M. Ghofrani, and M. Etezadi-Amoli, "Cost analysis of a power system using probabilistic optimal power flow with energy storage integration and wind generation," Electrical Power \& Energy Systems, vol. 53, Dec. 2013.

[21] T. Kerdphol, Y. Qudaih, and Y. Mitani, "Battery Energy Storage System Size Optimization in Microgrid using Particle Swarm Optimization," in 5th IEEE PES Innovative Smart Grid Technologies Europe (ISGT Europe), Istanbul, Oct. 2014. 
[22] X. Li, D. Hui, and X. Lai, "Battery Energy Storage Station (BESS)-Based Smoothing Control of Photovoltaic (PV) and Wind Power Generation Fluctuations," IEEE Trans. Sustain. Energy, vol. 4, no. 2, pp. 464-473, Apr. 2013.

[23] A. S. A. Awad, T. H. M. EL-Fouly, M. M. A. Salama, "Optimal ESS Allocation and Load Shedding for Improving Distribution System Reliability," IEEE Trans. Smart Grid, vol. 5, no. 5, pp. 2339-2349, Sept. 2014.

[24] M. Nick, R. Cherkaoui, and M. Paolone, "Optimal siting and sizing of distributed energy storage systems via alternating direction method of multipliers," International Journal of Electrical Power \& Energy Systems, vol. 72, pp. 33-39, Nov. 2015.

[25] M. Nick, M. Hohmann, R. Cherkaoui, and M. Paolone, "On the Optimal Placement of Distributed Storage Systems for Voltage Control in Active Distribution Networks," in 3rd IEEE PES Innovative Smart Grid Technologies Europe (ISGT Europe), Berlin, 2012.

[26] M. Nick, R. Cherkaoui, and M. Paolon, "Optimal Allocation of Dispersed Energy Storage Systems in Active Distribution Networks for Energy Balance and Grid Support," IEEE Trans. Power Syst., vol. 29, no. 5, pp. 2300-2310, Sep. 2014.

[27] S. Teleke, M. Baran, S. Bhattacharya, A. Huang, "Optimal control of battery energy storage for wind farm dispatching," IEEE Trans. Energy Convers., vol. 25, no. 3, pp. 787-794, Sep. 2010.

[28] A. Keyhani, Design of Smart Power Grid Renewable Energy Systems, Wiley-IEEE Press, 2011.

[29] F. Milano, "Power System Analysis Toolbox, Documentation for PSAT," User's Manual, 2008.

[30] K. E. Brenan, S. L. Campbell, and L. R. Petzold, Numerical Solution of Initial-Value Problems in Differential-Algebraic Equations, Society for Industrial and Applied Mathematics, 1995.

[31] S. A. Kalogirou, "Applications of artificial neural-networks for energy systems," Applied Energy, vol. 67, pp. 17-35, 2000.

[32] S. A. Kalogirou, "Artificial neural networks in renewable energy systems applications: a review," Renewable and Sustainable Energy Reviews, vol. 5, pp. 373-401, 2001.

[33] A. Hirose, Complex-Valued Neural Networks: Advances and Applications, IEEE Press Series on Computational Intelligence, 2013.

[34] M. Motalleb, S. Sreedarsan, and R. Ghorbani, "Power Grid Reliability Improvement through Forecasting with ComplexValued Neural Networks under System Contingency," Neural Networks, (Under Review).

[35] N. Zagoras, "Battery Energy Storage System (BESS): A Cost/Benefit Analysis for a PV power station," Clemson University Restoration Institute, Sep. 2014.

[36] E. Reihani, M. Motalleb , R. Ghorbani, and L. S. Saoud, "Load Peak Shaving and Power Smoothing of a Distribution Grid with High Renewable Energy Penetration," Renewable Energy, vol. 86, p. 1372-1379, Feb. 2016.

[37] E. Reihani and R. Ghorbani, "Load Commitment of Distribution Grid with High Penetration of Photovoltaics (PV) Using Hybrid Series-Parallel Prediction Algorithm and Storage," Electric Power Systems Research, vol. 131, p. 224-230, Feb. 2015.

[38] E. Reihani, S. Sepasi, L. R. Roose,and M. Matsuura, "Energy management at the distribution grid using a Battery Energy Storage System (BESS)," International Journal of Electrical Power \& Energy Systems, vol. 77, p. 337-344, 2016.

[39] "Hawaiian Electric Energy Cost Adjustment Factor for July 2015," K. Matsuura, Hawaiian Electric Company, Inc. 